\title{
Improving Activity Estimation in Passive Gamma Scanning for Radioactive Waste Drums
}

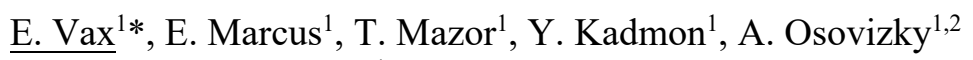 \\ ${ }^{1} \mathrm{NRCN}$, Israel \\ 2 Rotem Ind., Israel \\ * vax.eran@gmail.com
}

\begin{abstract}
A method to improve radioactive waste drum activity estimation in Segmented Gamma Scanning (SGS) systems was developed for homogenous content. We describe a method to quantify the activity of spatially distributed gamma-emitting isotopes ('hot spots') in homogenous content waste drums without the use of a collimator. Instead of averaging all the detector's readings we treat it as many different spatial samples as if we have multiple detectors surrounding the waste drum ("virtual detectors"). From these readings, we form a general linear model. Next, we derive the Maximum Likelihood Estimator (MLE) for the multiple sources position and activity. We solve this hyper-dimensional search problem using an Alternating Projections (AP) technique which transforms the problem into a simpler one-dimensional maximization problem. We tested this method using a mathematical simulation with a various number of sources, at random activities and positions for several energy bands. The preliminary results are consistent and show large improvement of the accuracy with comparison to industrial SGS systems and the same accuracy as new methods which exploits the spatial samples. Furthermore, since this method eliminates the need for heavy led collimator, none of the sources is blocked for the whole measurement period, which provides increased count rates and decreases the total measurement time.
\end{abstract}

Keywords-Radioactive waste drum, Alternating Projections, Projection algorithms, Maximum likelihood estimation, Passive scanning.

\section{INTRODUCTION}

G HIS Gamma-ray spectrometry uses the fact that each isotope emission gamma-rays in proportion to the mass of that isotope in the sample. However, not all emitted radiation will be detected by the detector. This is caused due to absorption and dispersion of the photons in the materials in its path to the detector, and in the source itself (self-absorption). Another effect is the energy efficiency of the detector. In Classical SGS some of these effects may be corrected with efficiency and transmission calibrations. In order to estimate the matrix density, many systems use a preliminary step of using an RTR system (real-time radiography) with X-ray, known external gamma or neutron source.

A standard SGS procedure for more accurate materials estimation is Isotope Specific (Mass) Calibration, which is the original SGS procedure, uses standards of known radionuclide masses to determine detector response in a mass versus corrected count rate calibration and [2] Efficiency Curve Calibration, typically uses non-SNM radionuclide sources to determine system detection efficiency vs. gamma energy. In this paper, the activity estimated is relative to the radiation flux reaching the detector and do not take into account the detector efficiency nor translate the counts to the mass of the radiating material.

Assaying waste drums that have a heterogeneous matrix and that contain a non-uniform radionuclide distribution is usually done with Tomographic Gamma Scanning (TGS) rather than SGS systems. However, when the matrix is homogenous with known density SGS can be adequate, especially after calibration.

In the classic Segmented Gamma Scanning (SGS) method the 208-liter drum is rotated in order to smooth spatial distributions of the activity and the matrix. The SGS method assumes a uniform activity distribution for each segment visible to the detector through a slit collimator. However, the waste activity distribution in the drum is concentrated at 'hot-spots'. The different 'hot spots' distances from the center of the drum are the main reason for wide reading variations when the drum is rotated. The different positions of the 'hot spots' are the main cause of activity estimation errors [1]. The first standard correction procedure handles gamma attenuation using density estimation. Next, the counts are accumulated while the drum is turning, in order to average the source distance from the detector. To minimize the reading variations the distance of the detector from the drum is increased. For many cases, this procedure gives adequate results, but in some cases the accuracy is poor, depending on the spatial distribution of the hot-spots in the drum. Industrial SGS systems for estimating the activity of 208-liter standard waste drums, declare on accuracy of $10-30 \%$ but with the worst case of up to $500 \%$ mistake [2]. A Recent method developed by [3] exploits the angular dependent count rate distribution and improved by [4], but requires long measurement time to get good counting statistics for the analysis. 
The goal of this work is to improve the accuracy results while reducing the total measurement time. To increase the count rate, we remove the narrow physical collimator and apply a digital filter on the measurements from the different spatial samples from the virtual detectors array surrounding the waste drum.

\section{METHOD}

\section{A. General Description}

In this paper, we describe a method to quantify the activity of spatially distributed gamma-emitting isotopes ('hot spots') in homogenous content waste drums without the use of a detector collimator. We use two steps to estimate the total activity in the drum. First, instead of minimizing the reading variations, the variations are exploited to localize the 'hot spots' with Maximum Likelihood Estimator (MLE) for multiple sources. Next, we calculate the activity based on the estimated hot spots positions using Least Squares (LS).

The ML estimator for the location of the sources, shown on part $\mathrm{B}$, is a type of a Beam Former (BF), taken from antennas array spatial signal processing [5]. However, solving this multidimensional maximization requires to search for $M$ sources in $\mathrm{K}$ discrete optional positions volume, which accumulate to $\frac{\mathrm{K} !}{\mathrm{M} ! \cdot(\mathrm{K}-\mathrm{M}) !} \stackrel{\mathrm{K} \gg>\mathrm{M}}{\longrightarrow} \mathrm{K}^{\mathrm{M}}$ search options. A nonoptimal but fast way is to develop the ML for a single source and then do a search in the drum volume and picking the $\mathrm{M}$ largest local maximums of the Likelihood. As a good compromise between the two options, we use an iterative technique referred to as "Alternating Projection" (AP) [6]. The AP technique, described in part D, is not optimal but performs better than the $\mathrm{M}$ single sources search.

\section{B. Problem formulation}

Under the assumption of known homogenous absorbing material, we define the attenuation factor $a_{n m}(\gamma)$, for the total attenuation between the $m$-th source in the drum, with activity $I_{m}$, to the counts in the $n$-th detector position surrounding the drum envelope for energy $\gamma$ (referred to as the $n$-th detector). The counts at detector $n$ originated from source $\boldsymbol{m}$ is marked as $y_{n m}(\gamma)$ :

$$
\begin{aligned}
y_{n m}(\gamma)= & a_{n m}(\gamma) \cdot I_{m}(\gamma) \quad \text { where } \\
& a_{n m}(\gamma)=\frac{1}{L_{n m}{ }^{2}} \cdot e^{-\mu_{i}(\gamma) \cdot l_{i n m}} \cdot e^{-\mu_{w}(\gamma) \cdot l_{w_{n m}}}
\end{aligned}
$$

$L_{n m}$ is the distance between source $n$ to detector $m$. the distance is comprised of $l_{i_{n m}}$ and $l_{w_{n m}}$, the partial distances the photons travel inside the drum, and crossing the drum wall respectively, the rest of the photon path through the air is ignored due to the small attenuation over the air. The attenuation coefficient inside the drum, and of the drum wall are marked $\mu_{i}(\gamma)$ and $\mu_{w}(\gamma)$. In vector form the total counts in the detectors are:

$$
\vec{y}_{t_{i}}(\gamma)=A(\gamma, D, S) \cdot \vec{I}(\gamma)+\vec{w}_{t_{i}}(\gamma), \quad t_{i}=1, \ldots T
$$

Where $\vec{y}_{j}(\gamma)=\left[\boldsymbol{y}_{\mathbf{1}}(\gamma), \ldots, \boldsymbol{y}_{\boldsymbol{N}}(\gamma)\right]_{j}^{T}$ is the vector of the $\mathrm{N}$ detectors readings, with contribution from all $M$ sources in each detector at energy $\gamma$, at time $t_{\mathrm{i}}$ and $\vec{I}(\gamma)=\left[\boldsymbol{I}_{\mathbf{1}}(\gamma), \ldots, \boldsymbol{I}_{\boldsymbol{M}}(\gamma)\right]^{T}$ is the $M$ sources activity vector. The noise $\vec{w}_{\mathrm{t}_{\mathrm{i}}}(\gamma)$ is independent identical additive white Gaussian noise vector and
$A(\gamma, D, S)$ is the attenuation coefficients matrix as defined in [2]. Matrixes $D$ and $S$ are the detectors and sources 3dimensional coordinates, size $3 \mathrm{xN}$ and $3 \mathrm{xM}$ respectively.

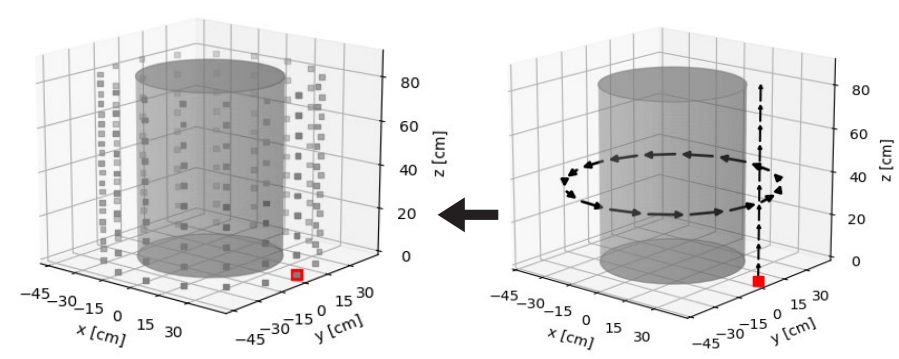

Fig. 1. Translating the rotation and upward motion into $\mathrm{N}$ spatial sample positions ('virtual detectors')

\section{Maximum Likelihood Estimator}

From the linear model we derive the Maximum Likelihood Estimator (MLE) of the multiple sources position (marked as $\left.S_{M}\right)$ and activities $\left(\vec{I}_{M}\right)$.

$$
L L=\log f_{\vec{y} / I, \vec{s}}\left(\vec{y} / \vec{I}_{M}, S_{M}\right) \underset{\vec{I}_{M}, S_{M}}{\longrightarrow} \max
$$

Under the assumption of knows sources positions $(\hat{S})$ we get the estimator for the sources activities:

$$
\hat{\vec{I}}(\gamma)=\left(A^{T} \cdot A\right)^{-1} \cdot A^{T} \cdot \vec{y}(\gamma)
$$

We reinstate the estimated activity into the log-likelihood and get:

$$
L L(\gamma, D, \hat{S})=\frac{J}{\sigma^{2}} \cdot \operatorname{tr}\left(P_{A}(\gamma, D, \hat{S}) \cdot \hat{R}_{y}\right)
$$

where $P_{A}$ is the projection matrix to the space spanned by the $M$ attenuation vectors and $\hat{R}_{y}$ is the sample covariance matrix calculated as:

$$
\begin{gathered}
P_{A}(\gamma, D, \hat{S})=A \cdot\left(A^{T} \cdot A\right)^{-1} \cdot A^{T} \\
\hat{R}_{y}=\frac{1}{J} \sum_{j=1}^{J}\left(\vec{y}-\frac{1}{J} \sum_{j=1}^{J} \vec{y}\right) \cdot\left(\vec{y}-\frac{1}{J} \sum_{j=1}^{J} \vec{y}\right)^{T}
\end{gathered}
$$

For a case with only a single source, the $L L$ is reduced to:

$$
\hat{\vec{s}}_{m l}=\arg \max _{\vec{s}}\left\{\frac{\vec{a}^{T}(\vec{s}) \hat{R}_{y} \vec{a}(\vec{s})}{\|\vec{a}(\vec{s})\|^{2}}\right\}
$$

Pay attention that there is no constraint on the sources activities to be positive in [5] since our final goal is not estimating each source activity but the total activity in the drum. This will be demonstrated with the example in the results section.

\section{Alternating Projections}

The ML solution is to search in the drum space for the source coordinate that maximizes the LL expression. In the single source, it is easily done by calculating $\mathrm{K}$ uniformly spread positions and finding the maximum. However, in the general $M$ sources problem, we need to do a 3-dimensional search of the $M$ optimal positions that maximize the $L L$ expression which is a resource and time-consuming task.

We solve this hyper-dimensional search problem for Maximum Likelihood localization of multiple sources using an Alternating Projections (AP) technique introduced by [6]. The AP has two steps. In the first step, we add source every search and keep the previous sources in fixed positions until we reach $M$ sources. 
On the second step we eliminate a random source, then we redo the search for the deleted source position while keeping the other M-1 sources fixed.

We start the first step by searching for a single source using [8] over the different source positions by changing the attenuation vector $\vec{a}(\vec{s})$. After finding the first source position $\vec{s}_{1}$, we search for the second source using [7], where the attenuation matrix A is built from two attenuation vectors - the first vector is from the position we found in the previous search, $\vec{a}\left(\hat{\vec{s}}_{1}\right)$, and we search over the possible positions of the second source attenuation vector. In the same manner, we repeat the search for the m-th source, by searching only for one source every time and keep the first $\mathrm{m}-1$ sources positions fixed, e.g. $A=\left[\vec{a}\left(\hat{\vec{s}}_{1}\right), \vec{a}\left(\hat{\vec{s}}_{2}\right), \ldots, \vec{a}\left(\hat{\vec{s}}_{3}\right), \vec{a}\left(\vec{s}_{m}\right)\right]$. After every step, we calculate the sources activities using [5]. Stopping condition for adding more sources is when the change in the sources activities is small.

The second step purpose is to refine the M sources positions estimation. We randomly pick one source and delete it. Then we search for a new source position with all the rest of the positions fixed. Again, the stopping condition is as before when the change of the positions and activities in each iteration is very small.

\section{PRELIMINARY RESULTS}

Mathematical simulation enabled to easily create the detectors reading vectors for many different configurations. These configurations had variable detectors spatial sample grid and different attenuations. Multiple simulations were executed for each configuration with variable radioactive sources quantity, activity and distribution.

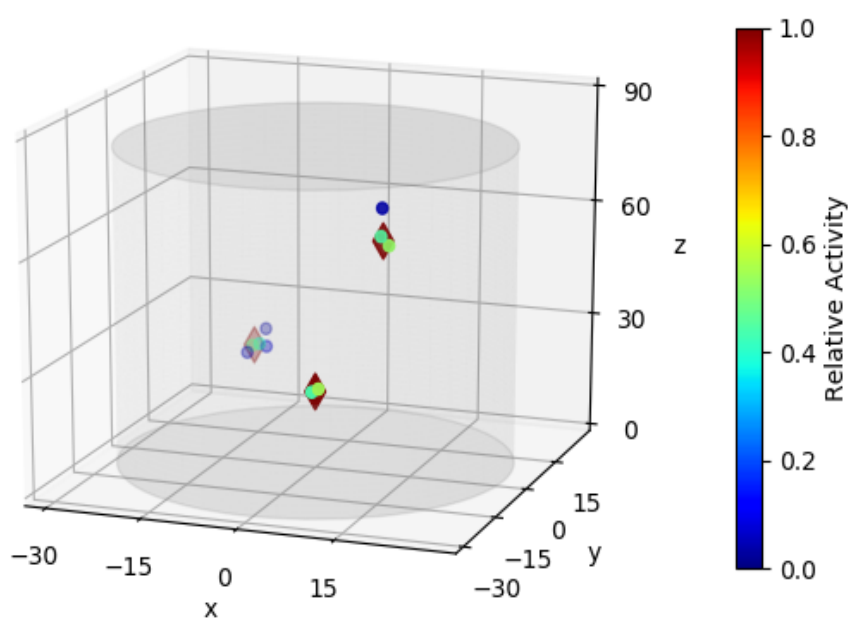

Fig. 2. Example of location and activity estimation for 3 sources with unit relative activity (red diamonds), using 10 sources (colored dots).

Figure 2 shows an example of the estimation result for simulation of 3 random positioned sources (blue diamonds), each with equal activity normalized to 1 . The estimation was made using a preset of 10 sources. The plot shows that each source was estimated using two, very close, sources with approximately half activity each (orange dots), while the other further four sources activity is close to zero (green dots). Notice that although there is no constraint on the sources to be positive, there are no large negative sources only small sources that are close to zero, which are compensation for the measurement noise, since the true sources were estimated very closely in position and activity.

\section{DISCUSSION AND CONCLUSIONS}

Traditionally, when designing a facility for LLW there are two main options - SGS or TGS. This work has shown a new type of system which has the advantage of simple mechanics, no need for heavy shielding, thus having higher efficiency and a shorter total time of measurement. From preliminary simulations results, it shows potential to be located between SGS and TGS in terms of activity estimation accuracy.

The first challenge was importing a technique which uses the phase difference in the arriving of an electrical field to antennas, to the radiation detectors which only measures the radiation field intensity. Next, we needed to find a way to dramatically reduce the $\mathrm{K}^{\mathrm{M}}$ searches to find $\mathrm{M}$ sources in $K$ discrete optional positions inside the drum volume. This was done using the AP technique. Last we needed to find a way to estimate roughly the number of hot-spots inside the drum. For this, there are a few estimation methods that were tested such as

Based on our preliminary results, we predict that a large improvement in the accuracy of comparing to the standard SGS systems can be expected. The biggest advantage of the suggested system is the lack of necessity for heavy led collimator. Since no source is blocked by the collimator, the count rates are increased and the total measurement time decrease.

\section{References}

[1] Dung, Tran Quoc. Calculation of the systematic error and correction factors in gamma waste assay system. Ann. Nucl. Energy, Vol. 24, No1. 1997, Vol. 24, 1, pp. 33-47.

[2] ORTEC. Comparison of Gamma-Ray Nondestructive Assay Measurement Techniques. s.l. : ORTEC, ANTECH Corp.

[3] "An improved method for the non-destructive characterization of radioactive waste by gamma scanning", Baia, Y.F., et al. 2009, Applied Radiation and Isotopes 67, pp. 1897-1903.

[4] "Reconstruction of the activity of point sources for the accurate characterization of nuclear waste drums by segmented gamma scanning", Krings, Thomas and Mauerhofer, Eric. 2011, Applied Radiation and Isotopes 69, pp. 880-889.

[5] Trees, H. L. van., "Arrays and Spatial Filters" in Optimum array processing - Part IV of detection, estimation, and modulation theory, John Wiley \& Sons, 2004.

[6] "Maximum Likelihood Localiztion of Multiple Sources by Alternating Projection", Ziskind, I. and Wax, M. ASSP-36, 1988, IEEE Trans.on ASSP, pp. 1553-1560.

[7] Group N-1. Segmented Gamma-Ray Scanner. Group N-1, Los Alamos National Laboratory. s.1. : Los Alamos National Laboratory, 1991. Application Note. LALP-91-011.

[8] Standard Test Method for Nondestructive Assay of Special Nuclear Material in Low-Density Scrap and Waste by Segmented Passive Gamma-Ray Scanning. s.l.: ASTM International, 2018. C1133. 